\title{
Unternehmerische Resilienz (nicht nur) in Krisenzeiten durch Corporate Integrity Controlling (CICO)
}

\section{Ausgangslage und Problemstellung}

Im Rahmen der Corona-Pandemie hat die weltweite Vernetzung von Wirtschaftssubjekten neue Konfliktfelder hervorgebracht, die sich medienwirksam vor allem durch die Beschlagnahmung von mehr als 200.000 Atemschutzmasken und gefälschten sowie wirkungslosen Schutzkleidungsprodukten und Desinfektionsmitteln manifestiert haben (vgl. ntv 2020a und 2020b). Dieses wie auch weitere Beispiele jener Art zeigen, dass heutige Supply-Chain-Beziehungen erhöhten Ansprüchen unterliegen, die von unterschiedlichsten Stakeholdern mitbestimmt werden. Dazu gehören nicht nur Aspekte der Sicherheit und der Verlässlichkeit innerhalb von Lieferantenbeziehungen, sondern auch die Beachtung und Einhaltung einer allgemeingültigen Integrität, die u. a. auch durch Kriterien wie Nachhaltigkeit und einer umfassenden Corporate Social Responsibility (CSR) geprägt ist.

Wenngleich in den meisten Unternehmen heute die Absicht besteht, Lieferketten transparent und in Bezug auf die gegenseitigen Geschäftsbeziehungen integer zu gestalten, so offenbaren die genannten Vorfälle in Krisensituationen die mangelnde Resilienz der Organisationen gegenüber schädlichen bzw. unethischen Geschäftspraktiken.

Die Unsicherheit auf den Märkten und neue, krisenbedingte Prioritätensetzungen sowie staatliche Eingriffe in Lieferketten stellen nicht nur Unternehmen, sondern auch ganze Gesellschaften vor ein bisher kaum wahrgenommenes ethisches Problem, das in dem Aufbau von Vermögenswerten zu sehen ist, die einen vertrauensbasierten Charakter aufweisen (vgl. Suchanek/von Brook 2020) und Zuverlässigkeit sowie Loyalität innerhalb der Supply-Chain garantieren sollen. Kurzfristige Entscheidungen und schnelle Handlungen können nur dann erfolgreich realisiert werden, wenn Beziehungen bereits zuvor integer gestaltet wurden und damit resilient gegenüber >Angriffen von außen`sind. Ad-hoc-Kooperationen, wie sie in Krisenzeiten nahezu täglich im privaten, gesellschaftlichen und vor allem geschäftlichen Umfeld geschehen, basieren auf Vertrauen, das seine Grundlage in einem Attributionsprozess auf der Basis wahrgenommener Integrität des jeweiligen Partners erfährt (vgl. Gröppel-Klein et al. 2009: 301).

Problematisch ist es jedoch, wenn auch in Krisenzeiten die Tendenz zur Profitmaximierung bestehen bleibt. Es scheint, als ob gerade im Rahmen von Lieferketten bei der Wahl der Supplier dann über die Verletzung und Ignoranz ethischer Werte hinweggesehen wird, wenn das eigene Überleben durch diese Geschäftsbeziehung gesichert werden kann. In seinen Langzeitfolgen führt dies zu erheblichen 
Vertrauensverlusten, die im Hinblick auf gesellschaftliche Anspruchsgruppen die Gefahr des Verlustes von Reputationskapital implizieren. Besonders deutlich wird diese Störanfälligkeit im Kontext des Supply-Chain-Managements, da es in seiner rechtlichen wie auch organisatorischen Gestaltung nicht nur auf individuellen Einzelabsprachen und Verträgen zwischen den Partnern basiert, sondern häufig auch aufgrund kulturell bedingter Auslegungen der Gefahr unterschiedlicher Interpretationen unterliegt. Dies hat zu einer gesteigerten $>$ Normsetzung durch Auslegung (Rüthers 2014: 148) und zu großen Unwägbarkeiten innerhalb von Geschäftsbeziehungen, aber auch der Gesellschaft allgemein geführt (vgl. Luhmann 2002: 50; Rüthers 2014: 145) und eröffnet damit Unternehmen und sogar einzelnen Staaten ein ungeahnt weites Feld für Neu-Interpretationen getroffener Absprachen im Sinne eines auf Eigeninteressen fokussierten Ziels im Kontext kultureller Eigenschaften oder regional bedingter Interessen.

\section{Stand der Forschung: Integrität als Resilienzfaktor im Kontext des Supply Chain Managements}

\subsection{Corporate Social Responsibility als Rabmenbedingung}

Aus organisationaler wie aus gesellschaftlicher Perspektive hat die Integrität des Lieferantenmanagements (Supply Chain Management $=$ SCM) und damit streng genommen sogar die gesamte organisationale Integrität bisher kaum wissenschaftliche Betrachtung erfahren. Vor dem Hintergrund, dass im Rahmen einer globalisierten Welt die Unternehmen untereinander nicht nur Beziehungen aufbauen, sondern vor allem auch zur eigenen Absicherung bewahren müssen, ist dies als durchaus überraschend einzustufen. So zeigen bereits Josef Wieland und Michael Fürst, dass durch die Globalisierung einerseits größere Konfliktherde innerhalb von Lieferantenbeziehungen offenbart werden, wie z. B. die zunehmende Beachtung von Kinderarbeit, Arbeitsstandards allgemein, Gefangenenarbeit und Menschenrechtsfragen. Andererseits greifen auch die bisherigen rechtlichen Regelungen im Sinne einer einfachen Input-Output-Beziehung nicht mehr, da Lieferantenbeziehungen inzwischen einer eigenen Dynamik mit Dominanz in Bezug auf Zweckorientierung und Interessenabwägung unterliegen (vgl. Wieland/Fürst 2003: 2). In der vorhandenen Literatur zur Gestaltung von Supply-Chain-Systemen ist in der jüngsten Vergangenheit besonders der Faktor der Corporate Social Responsibility diskutiert worden. Dabei geht es inzwischen weniger um die Frage, ob unternehmerisches Handeln CSR-konform ausgerichtet werden sollte, sondern vielmehr um dessen praktische Umsetzung (vgl. Searcy 2009: 1057). Ein verantwortlicher Umgang mit Ressourcen sowie eine gesellschaftlich-nachhaltige Geschäftstätigkeit waren auch in der Vergangenheit bereits in Form von Werten, Traditionen und Moral Bestandteile des Supply-Chain-Managements, die immer dann besonderen Belastungen ausgesetzt sind, wenn sich Märkte und Strukturen innerhalb kürzester Zeit plötzlich verändern und Unternehmen einem sehr hohen 
Anpassungsdruck ausgesetzt sind. Die Existenz neuer Stakeholder wie auch nationaler Gesetzgebungen verlangt von den Unternehmen eine erhöhte Adaptionsfähigkeit (vgl. Dahlsrud 2008: 6; Karaibrahimoglu 2010: 383), was sich in SupplyChain-Beziehungen vor allem unter den Bedingungen der Corona-Krise deutlich gezeigt hat. Die strategische Ausrichtung von SC-Beziehungen wird somit nicht nur durch die Weiterentwicklung von CSR-Konzepten beeinflusst, was insbesondere durch die Vereinten Nationen im Rahmen der 2030-Agenda aufgegriffen wurde (vgl. United Nations 2020), sondern muss auch unter spontanen Vorkommnissen wie politischen Entscheidungen, das Auftreten von Skandalen oder eben auch die Corona-Pandemie ihre Stabilität unter Beweis stellen (vgl. Fröhlich et al. 2011: 9). Damit verbundene Aspekte wie Moral, Fairness und Vertrauen verfolgen viele Unternehmen bisher lediglich als vereinzelte Substrategien innerhalb ihrer Supply-Chain-Strukturen, um damit den Erwartungen ihrer Stakeholder zu entsprechen, aber weniger, um damit ein eigenes ethisches Fundament der Unternehmensführung aufzubauen (vgl. Jonker et al. 2011: 40). Integrität, verstanden als gesamtorganisatorische Ausrichtung, kann vor diesem Hintergrund einen wesentlichen Beitrag dazu leisten, Geschäftsbeziehungen im Sinne einer konfuzianischen Dynamik (langfristige Orientierung, basierend auf Werten wie Fleiß, Sparsamkeit und Zielstrebigkeit, indem Unternehmensziele in die Zukunft projiziert und angestrebt werden) dauerhaft zu stabilisieren und auch in Krisenzeiten die Organisation vor Angriffen von außen zu schützen.

\subsection{Organisationale Integrität als Resilienzfaktor in Krisenzeiten}

Verantwortliches und stakeholderkonformes Verhalten wird in den meisten Organisationen heute mit einer Beachtung sogenannter CSR-Richtlinien gleichgesetzt. Mehr als 80 Prozent aller Fortune-500-Unternehmen bekennen sich auf ihren Webseiten zu CSR (vgl. Sen/Bhattacharya 2001: 225). Allerdings zeigt die Praxis, dass eine Integration in die Unternehmensstrategie sowie in einzelne Funktionsbereiche noch recht zögerlich erfolgt (vgl. Fröhlich et al. 2011: 9; Schneider 2012: 17). Gerade in Lieferketten macht sich dieses Manko bemerkbar, da diese von Aspekten wie Dauer der Beziehung, Leistungsvolumen und vor allem informationstechnischen Kommunikationsstandards beeinflusst werden, sodass aus der organisationalen Betrachtung heraus ein besonderes Beziehungsmanagement erforderlich ist (vgl. Christopher 1999: 18). Hieraus ergeben sich zugleich mehrere Problemstellungen. Diese fokussieren in erster Linie den Zusammenhang zwischen der Dichte einer Beziehung und dem ökonomischen Erfolg der beteiligten Partner sowie die Frage nach möglicherweise zu etablierenden Kontrollstrukturen, die die beteiligten Unternehmen vor opportunistischen Verhaltensweisen ihrer jeweiligen Geschäftspartner schützen. 


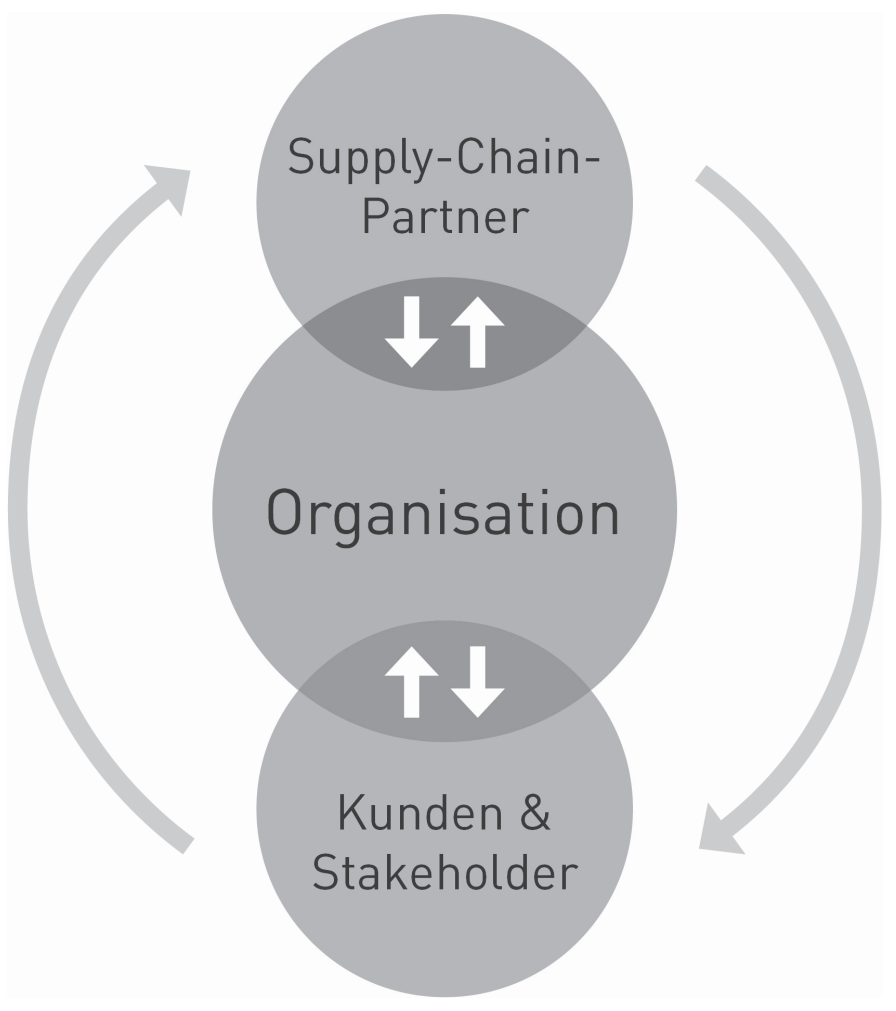

Abbildung 1: Beziehungen im Supply-Chain-Management unter Aspekten der Integrität (Quelle: eigene Darstellung)

Zwar offerieren Compliance-Programme und daran anhängige Vereinbarungen eine vermeintliche Absicherung gegenüber internen und externen Einflüssen. Sie können jedoch auch negativ für die Geschäftsbeziehungen des Unternehmens sein, weil sie ein hohes Maß an Vertrauen voraussetzen, welches bei jungen Geschäftsbeziehungen oder wechselndem Personal ein Risiko darstellt. Sie stützen sich hauptsächlich auf allgemeine Aussagen und ethische Leitlinien, die nicht in Kennzahlensysteme überführt oder an die jeweilige Geschäftsstrategie angepasst worden sind. Bei der Anbahnung und Realisierung von Lieferantenbeziehungen und Geschäftspartnerschaften, vor allem wenn sie wie in Krisenzeiten schnell realisiert werden müssen, ist es aber auch essenziell, nach außen eine integritätsorientierte Lieferantenbewertung vorzunehmen, um die Orientierung an den jeweils vorhandenen Standards sowohl in die eine wie auch in die andere Richtung zu gewährleisten.

Die angestrebte Verknüpfung zwischen einer integren Lieferantenbeziehung und den daraus resultierenden betriebswirtschaftlichen Effekten im Sinne einer 
gesamtorganisationalen Integrität ist in Theorie und Praxis bisher nicht in Form eines strukturierten Kennzahlensystems vorhanden. Integrität unterliegt bisher einer eher individualethischen Betrachtung, was besonders daran erkennbar ist, dass mit dem Adjektiv sinteger in der Literatur vor allem menschliche, aber keine organisationsbezogenen Eigenschaften assoziiert werden (vgl. Maak/Ulrich 2007: 6-11). So zeigen z. B. Patrick Renz et al. (2014) erste Ansätze für eine Implementierung von Integrität im Managementalltag auf, fokussieren dabei aber die menschliche Einzelfallperspektive, z. B. im Rahmen von Führungsverhalten oder individuellen Prozessen in Organisationen, wobei auch nicht immer eine klare Abgrenzung zur Compliance erkennbar ist. Weitere Arbeiten unternehmen zwar einen ersten Versuch, Integrität auf organisationaler Ebene zu erfassen (vgl. z. B. Schöttl 2017), aber auch hier fehlen konkrete messbare Parameter, die organisationale Integrität eindeutig abbilden und international vergleichbar machen. Die individualethische Perspektive der Integrität erschließt sich zwar insofern sinnlogisch, als es natürlich immer Individuen sind, die für das jeweilige Unternehmen handeln. Gemäß dem Kulturidentifikationsprinzip nach Edward Heinen sollten diese aber den Werten und Prinzipien der jeweiligen Organisation folgen, sodass die eigentliche Integrität der Organisation den individuellen Prinzipien vorangestellt ist (vgl. Schöttl 2017: 69).

Die Implementierung eines integritätsüberprüfenden Controllinginstruments könnte vor diesem Hintergrund einen ersten Lösungsansatz für das vorhandene Spannungsfeld darstellen. Hierbei sollte es sich um einen integrativen Ansatz zur Verschmelzung der beiden Bereiche innere und äußere Integrität (= I) handeln, die sich sowohl mit Blick auf die gesamtgesellschaftlichen Anspruchsgruppen (Corporate $=\mathrm{C}$ ) und damit nach außen wie auch individual-betrieblich nach innen in eindeutige Kennzahlen (Controlling = $\mathrm{CO}$ ) übersetzen und in einen einzelnen unternehmensspezifischen Integritätsfaktor abbilden lassen (= CICO).

Da sich weltwirtschaftliche Umstände und organisationale Bedingungen gegenseitig beeinflussen und in Wechselwirkung zueinanderstehen, ist besonders der Faktor der Informationsasymmetrien sowohl in bestehenden als auch in sich anbahnenden Supply-Chain-Beziehungen entscheidend. Gerade in Krisenzeiten müssen Unternehmen teilweise ad hoc reagieren, Geschäftspartnern nahezu blind vertrauen, auf vielleicht wichtige Informationen aus Zeitgründen verzichten und sich auf Aussagen und Versprechen verlassen. Dies stellt nicht nur CSR-strategisch, sondern auch langfristig für das Unternehmen ein großes Risiko dar. Je länger und auch undurchsichtiger eine Supply-Chain ist, desto schwieriger wird es für die Protagonist*innen, Integrität zu garantieren und Informationen entlang der Lieferkette transparent zu halten. Daher stellt die Konzipierung eines messbaren, organisationsorientierten Integritätstools mit Blick auf die Planung, Steuerung und Kontrolle von Geschäftsbeziehungen eine unabdingbare Voraussetzung zur Gewährleistung und Pflege organisationaler Integrität dar, die Unternehmen und ihre Partner (nicht nur in Krisenzeiten) resilient gegenüber schädlichen Ein- 
flüssen von außen werden lässt. Der bisher konzeptionelle Ansatz >CICO< bietet dafür einen ersten Lösungsansatz an.

\section{CICO als konzeptioneller Ansatz zur Messung organisationaler Integrität}

\subsection{Hintergrund und vorgesehene Inhalte von CICO}

Erfolgreiches Supply-Chain-Management beruht auf dem Zusammenwirken von (digitaler) Technologie, realwirtschaftlichen Abläufen und den beteiligten Mitarbeiter"innen (vgl. Arora/Krause 2010: 225). Je internationaler und komplexer Supply-Chain-Ketten werden, umso mehr Verantwortung trägt das einzelne Unternehmen in Bezug auf die Herkunft und die Herstellung seiner Produkte und Komponenten und umso mehr unterliegt die Organisation dem Risiko mangelnder Integrität. Werden durch Geschäftsbeziehungen individuelle Mission Statements oder Werterahmen sowie generell der Anspruch der unternehmerischen Integrität verletzt, unterliegt damit auch die gesamte Integrität der beteiligten Supply-Chain-Partner einem immensen Risiko (vgl. Renz et al. 2014: 58). Im Zweifel zeigt sie sich nicht resilient gegenüber potentiellen Störgrößen, wie sie aus der institutionenökonomischen Theorie bekannt sind (vgl. Voigt 2009: 13-17): z. B. das einseitige Ausnutzen eines Geschäftspartners durch unvollständige Verträge (Hold Up), vorvertragliche Verschleierung von Eigenschaften und Qualifikationen als Auslöser für Adverse-Selection-Probleme (sogenannte >Hidden Characteristics<) oder auch die Nichtbeobachtbarkeit und mangelnde Zurechenbarkeit (`Hidden Action $<$ ) als Ursache für nachvertragliches `Shirking $>$ (>Moral Hazard $<$ ).

Um organisationale Integrität anhand konkreter Parameter messbar zu machen, ist es zunächst erforderlich, diese für sich zu definieren. Der Begriff der Integrität als solches unterliegt in der fachwissenschaftlichen Literatur Beschreibungen und Auffassungen, die in erster Linie die individualethische Komponente von Einzelpersonen fokussieren (vgl. Göbel 2013: 110). Im Hinblick auf organisationale Integrität liegen bisher nur wenige wissenschaftlich fundierte Definitionen vor. Patrick Renz et al. (2014) nähern sich zum Beispiel über eine Negativdefinition: Eine Institution ist nicht integer, wenn Spannungsfelder im Sinne von Inkonsistenzen existieren, und zwar bezüglich der Art, wie gearbeitet wird, und den Vorgaben, die die Organisation macht, und Werten, die sie hat. Werden diese Spannungsfelder nicht aufgegriffen und bearbeitet, existiert eine nicht integre Organisation (vgl. Renz et al. 2014: 23). Die Schwierigkeit der organisationalen Integrität ergibt sich also nicht nur durch die Wahrnehmung der unterschiedlichen Anspruchsgruppen in Bezug auf deren Ausgestaltung, sondern sie ist im besonderen Maße auch von dem eigenen moralisch konsistenten Verhalten des Unternehmens beeinflusst. Dies bedeutet, dass eine Organisation Integrität nicht einfach hat, sondern dass diese aus einer gelebten und gepflegten Wechselbeziehung mit anderen entsteht (vgl. Maak/Ulrich 2007: 6-11). Vor dem Hintergrund, dass $\mathrm{CICO}$ aber ein global einsatzfähiges, international allgemeingültiges System mit 
gleichem Integritätsverständnis abbilden soll, ist es unerlässlich, genauere, international vergleichbare Parameter von Integrität zu definieren sowie den Status quo der Lieferantenbeziehungen in Bezug auf den Stellenwert von Integrität zu erforschen. Daher wurde mit Blick auf die Ansprüche einer gesamtorganisationalen Integrität eine Metastudie auf Basis der Grounded Theory durchgeführt. ${ }^{1}$ Erste Ergebnisse dieser Studie zeigen, dass ein immenser Nachholbedarf in der objektiven und vor allem auf Kennzahlen basierenden Beurteilung von Lieferantenintegrität besteht. Im Ergebnis wird deutlich, dass weder eine systematische noch eine nachhaltige oder gar ethische Bewertung von Geschäftsbeziehungen vorgenommen wird, die einer vergleichbaren Anwendung folgt. Gleichwohl stimmen die befragten Unternehmen insofern überein, als für sie eine integre Geschäftsbeziehung in erster Linie die drei Parameter Störanfälligkeit (Resilienz), Transparenz (Abbau von Informationsasymmetrien) und Authentizität der jeweiligen Partner die entscheidenden Kriterien darstellen, die in einem international vergleichbaren Integritätsfaktor abgebildet und operationalisiert werden sollten.

Der Integritätsfaktor CICO sieht daher zwei Scoringbereiche vor: Zum einen ermittelt er - basierend auf einem konkret dafür entwickelten digitalen Tool mit bereichskompatiblen und zugleich bereichsspezifischen Fragen - konkret quantifizierbare Parameter für die Sektoren `Resilienz‘, ’Transparenz` und `Authentizität` und errechnet auf Basis dessen einen Integritätswert (Gesamt-Scoring-Wert), der auf internationale Vergleichbarkeit ausgerichtet ist. Zum anderen soll er durch regelmäßige Wiederholungen in Form einer Überprüfung von bestehenden Partnerschaften eine stetige Risikobewertung vornehmen und so mögliche Interruptionen identifizieren, die die Supply Chain stören und ggf. sogar zu einem Abbruch führen könnten (z. B. Wechsel von Lieferantenpartner*innen). Darüber hinaus soll das Tool konkret ableitbare Maßnahmen und Vorschläge zur Verfügung stellen, die nicht nur zur Forderung und Förderung eines integreren Verhältnisses zwischen den SC-Partner dienen sollen, sondern auch durch eine entsprechende Kommunikation nach außen (`Dieser Betrieb ist CICO-zertifiziert $`$ ) die jeweiligen Unternehmen auch international als integer sichtbar werden lassen. Ferner ist vorgesehen, dass CICO die Dokumentation und Abbildung auffälliger Geschäftspraktiken vornimmt, die über den Zeitverlauf in längeren Beziehungen entstehen können, was somit dazu beiträgt, dass bestehende Supply-Chain-Allokationen regelmäßig evaluiert und neu bewertet werden können.

Durch die anhängigen Kennzahlen und den individuell abrufbaren Maßnahmenkatalog stellt CICO Lösungsmöglichkeiten zur Verfügung, wenn integritätstorpedierende Handlungen erfolgt sind oder das Risiko dessen besteht. CICO funktioniert daher wie ein Frühwarnsystem, das bei konsequenter Pflege Unternehmen auch im Falle von Rechtsstreitigkeiten, die ethische Aspekte zum Inhalt

1 Hierbei handelt es sich um eine Studie der Autorin, die bisher unveröffentlicht ist und sich noch teilweise in/derfinalen Auswertung befindet. 
haben, als Subjekt der Verantwortung absichern kann und über bisher bestehende Performance-Measurement-Systeme deutlich hinausgeht.

\subsection{Aktueller Entwicklungs- und Konzeptionsstand}

Die Entwicklung von CICO beruht gegenwärtig auf drei wesentlichen Forschungssträngen. Diese beinhalten:

1. Erforschung und Ableitung des Faktors >organisationale Integrität< in Bezug auf dessen definitorische Ausgestaltung, seiner Anwendung im Feld des Supply-Chain-Managements und dessen Wichtigkeit als unternehmerischer Resilienzfaktor (gerade in Krisenzeiten)

2. Analyse und Operationalisierung vorhandener und potenziell entstehender Informationsasymmetrien in Lieferantenbeziehungen unter den drei auf Basis von Metastudien identifizierten Integritätsparametern >Resilienz‘, Transparenz< und >Authentizität<

3. Ableitung sogenannter Interruptionsmechanismen, die vermeintlich integre Geschäftsbeziehungen stören und damit weniger resilient gegenüber Einflüssen von außen werden lassen

\section{Zusammenführung und Fazit}

Die Vorkommnisse der aktuellen Corona-Krise in Bezug auf intakte und vor allem nicht intakte Lieferantenpartnerschaften haben aufgezeigt, dass ein numerisches und damit objektivierendes Tool zur organisationalen Integritätsbewertung hilfreich und wünschenswert ist. Gerade außerhalb von Krisensituationen stellt es ein wichtiges Werkzeug zur Immunisierung der Protagonist*innen einer Supply-Chain gegen die üblichen Störfaktoren einer Lieferkette dar. Genau diese Immunisierung ist es, die als >Nebeneffekt <ür Notsituationen die dafür erforderliche Resilienz schaffen würde. Das Risiko, gerade neue Lieferantenpartner falsch einzuschätzen, kann durch eine objektivere Bewertung der gesamten Supply-Chain deutlich reduziert werden. CICO liefert dafür insofern Abhilfe, als durch die Erfassung konkreter Parameter und deren Abbildung in Form von skalierten Werten eine eher organisationale Dimension von Integrität wahrgenommen werden kann. Somit ermöglicht CICO eine neue Dimension der Integritätsbewertung und schützt Unternehmen wie auch Stakeholder zumindest in diesem Bereich vor schädigenden Fehleinschätzungen und -allokationen - ein langfristiger Vorteil, der sich besonders in Krisenzeiten bemerkbar macht und daher eine entsprechende Investition lohnt. 


\section{Literaturverzeichnis}

Arora, D./Krause, H.-U. (2010): Controlling-Kennzahlen - Key Performance Indicators. Zweisprachiges Handbuch Deutsch/Englisch - Bilingual Compendium German/English, München: Oldenbourg.

Christopher, M. (1999): Logistics and Supply Chain Management. Strategies for Reducing Cost and Improving Performance, London: Pearson Verlag.

Dahlsrud, A. (2008): How Corporate Social Responsibility is Defined: An Analysis of 37 Definitions, in: Corporate Social Responsibility and Environmental Management, Vol. 27/No. $3,1-13$.

Fröhlich, E./Weber, T./Willers, C. (2011): Nachhaltigkeit in der unternehmerischen Supply Chain, in: Fröhlich, E.; Weber, T.; Willers, C. (Hrsg.): Sammelband der Fördergesellschaft für Produktmarketing e. V., Berlin: ePubli.

Göbel, E. (2013) Unternehmensethik. Grundlagen und praktische Umsetzung, Stuttgart: Lucius \& Lucius Verlag.

Gröppel-Klein, A./Germelmann, C. C. (2009): Medien im Marketing. Optionen der Unternehmenskommunikation, Wiesbaden: Gabler Verlag.

Jonker, J./Stark, W./Tewes, S. (2011): Corporate Social Responsibility und nachhaltige Entwicklung, Berlin, Heidelberg, New York: Springer Verlag

Karaibrabimoglu, Y. Z. (2010): Corporate Social Responsibility in Times of Financial Crisis, in: African Journal of Business Management, Vol. 4/No. 4, 382-389.

Maak, T./Ulrich, P. (2007): Integre Unternehmensführung - ethisches Orientierungswissen für die Wirtschaftspraxis, Stuttgart: Schaeffer-Poeschel Verlag.

$n t v:$ (2020a) China konfisziert Millionen Schutzmasken. Link: https:/www.n-tv.de/panorama/ China-konfisziert-Millionen-Schutzmasken-article21740632.html (zuletzt abgerufen am 13. Juni 2020).

$n t v$ (2020b): USA fangen von Berlin bestellte Schutzmasken ab. Link: https://www.n-tv.de/polit ik/USA-fangen-von-Berlin-bestellte-Schutzmasken-ab-article21691173.html (zuletzt abgerufen am 14. Juni 2020).

Renz, P. S./Frischherz, B./Wettstein, I. (2014): Integrität im Managementalltag. Ethische Dilemmas im Managementalltag erfassen und lösen, Luzern, Berlin, Heidelberg: Springer Gabler Verlag.

Rüthers, B. (2014): Die heimliche Revolution vom Rechtsstaat zum Richterstaat - Verfassung und Methoden (Ein Essay), Tübingen, Mohr Siebeck.

Schöttl, L. (2017): Integrität in Unternehmen. Dissertation: Springer Fachmedien Wiesbaden. Link: https://books.google.com.pe/books?id=TGRaDwAAQBAJ (zuletzt abgerufen am: 24. Juli 2020).

Schneider, A. (2012): Reifegradmodell CSR. Eine Begriffserklärung und -abgrenzung, in: Schneider, A./Schmidpeter, R. (Hrsg.): Corporate Social Responsibility. Verantwortungsvolle Unternehmensführung in Theorie und Praxis, Berlin, Heidelberg: Springer-Verlag, $17-38$

Searcy, C. (2011): Corporate Sustainability Performance Measurement Systems: A Review and Research Agenda, in: Journal of Business Ethics, Vol. 107/No. 3.

Sen, S./Bhattacharya, C. B. (2001): Does Doing Good Always Lead to Doing Better? Consumer Reactions to Corporate Social Responsibility, in: Journal of Marketing Research, Vol. 38/No. 2, 225-243.

Smolenski, F. (2008): Controlling in internationalen Unternehmen, Wismarer Schriften zu Management und Recht - Bremen: Europ. Hochsch.-Verlag.

Suchanek, A./von Broock, M. (2020): COVID 19: In der Krise zählt Integrität - Vor allem für die Zeit danach! Link: https:/www.forum-wirtschaftsethik.de/covid-19-in-der-krise-zaehlt -integritaet-vor-allem-fuer-die-zeit-danach/ (zuletzt abgerufen am 13. Juni 2020). 
United Nations (2020): Making Global Goals Local Business. Link: https://www.unglobalcom pact.org/sdgs (last access on June 13th, 2020).

Voigt, S. (2009): Institutionenökonomik, Wien: UTB Verlag

Wieland, J./Fürst, M. (2003): Integrität und Wertemanagement in der Lieferantenbeziehung. Zur Operationalisierung eines integritätsorientierten Lieferantenbewertungssystems, KIeM Working Paper, No. 05/2003. 